\title{
Thrombotic thrombocytopenic purpura as the first presentation in systemic lupus erythematosus
}

\author{
Opeyemi Folorunsho Bamidele ${ }^{1}$, Richard Oluyinka Akintayo ${ }^{1}$, Mathew Olumuyiwa Bojuwoye ${ }^{1}$, \\ Tolulope Oyetunde Alabi ${ }^{1}$, Foluke Comfort Akintayo ${ }^{2}$, Oluwakemi Victoria Bamidele ${ }^{1}$ \\ ${ }^{1}$ Department of Medicine, University of Ilorin Teaching Hospital, Ilorin, Nigeria \\ ${ }^{2}$ Department of Family Medicine, Ladoke Akintola University Teaching Hospital, Ogbomoso, Nigeria
}

\begin{abstract}
Thrombotic thrombocytopenic purpura (TTP) is an uncommon, life-threatening disease characterized by severe thrombocytopenia, microangiopathic haemolytic anaemia, neurologic abnormalities, renal insufficiency, and fever commonly associated with infections, malignancy, drugs, and autoimmune diseases. Coexistence of TTP with systemic lupus erythematosus (SLE) is extremely rare, and the emergence of the full manifestation of SLE starting with TTP is even rarer. Black people due to genetic risk factors are at increased risk of TTP. In the present article the authors describe the case of a Nigerian woman who developed classical features of TTP as the first manifestation of SLE. The patient's condition was stabilized after treatment with intravenous pulses of methylprednisolone and subsequently on oral prednisolone and hydroxychloroquine treatment.
\end{abstract}

Key words: thrombotic thrombocytopenic purpura, systemic lupus erythematosus, Nigerian.

\section{Introduction}

Systemic lupus erythematosus (SLE) is an autoimmune multi-systemic disease characterized by the presence of autoantibodies directed against nuclear antigens [1]. Patients can present in vastly different ways, with polyarthralgia, fever, skin photosensitivity with malar or butterfly rush and hair loss as the most common presentation, and in severe disease SLE may cause exudations in serous membranes, nephritis, central nervous system involvement, leukopenia, autoimmune haemolytic anaemia and thrombocytopenia [2]. Almost all patients with SLE have positive anti-nuclear antibodies (ANA) at significant titres of $1: 80$ or greater and presence of anti-double-stranded DNA (anti-dsDNA) and anti-Smith (anti-SmAb) antibodies [1].

Thrombotic thrombocytopenic purpura (TTP) is a life-threatening disorder with characteristic von Willebrand factor-rich microthrombi (VWF) affecting the arterioles and capillaries of multiple organs caused by deficiency of plasma metalloprotease ADAMTS13, which is well known as von Willebrand factor-cleaving prote- ase [3]. Thrombotic thrombocytopenic purpura can be hereditary through mutation of the ADAMTS13 gene or through autoimmune inhibitors of ADAMTS13 in acquired cases such as SLE, infections and neoplasms. Thrombotic thrombocytopenic purpura in patients with SLE is extremely rare, with an incidence as low as $0.5 \%$ among this group of patients [4]. Interestingly, connective tissue diseases, including SLE, may proceed with low levels of ADAMTS13, suggesting a possible pathophysiology for coexistence of these two diseases [5].

\section{Case report}

A 58-year-old Nigerian female patient was admitted to the emergency unit due to watery stools for several days and bilateral knee pain of three-day duration. The volume of watery stool was estimated to be about 250$300 \mathrm{ml}$ per episode; it was occasionally blood stained but not mucoid. Additional symptoms were crampy lower abdominal pain, fever and generalized weakness.

About a day prior to admission the patient became drowsy, not responding to voice, without seizures, head- 
ache, vomiting or bleeding from craniofacial orifices. The knee joints were painful and swollen.

Physical examination revealed pale skin with widespread purpura and ecchymosis on the upper and lower extremities and the trunk, mild dehydration, and fever (not exceeding $38^{\circ} \mathrm{C}$ ). There was no peripheral oedema or cyanosis. In the neurological examination, the patient was assessed on the Glasgow Coma Scale as 13 points out of 15. There was no sign of meningeal irritation, and no disturbances of superficial or deep sensations were observed. The muscle tension and reflexes were normal and there was no focal neurologic deficit. In the examination of the musculoskeletal system both knees were warm, tender and swollen. The abdomen was full and moved with respiration. The intra-abdominal organs were not palpably enlarged and there were no symptoms of ascites. The respiratory and cardiovascular examinations were not remarkable.

The platelet count was decreased: $59 \times 10^{9} /$ I (reference: $150-450 \times 10^{9} /$ l); packed cell volume (PCV), 23\% (reference: 35-45\%); and white blood cell count, $9.4 \times 10^{9}$ (reference: 4-12 $\left.\times 10^{9}\right)$. Peripheral blood showed numerous schistocytes greater than 3\% (reference: $0.5 \%$ ). Serum creatinine was $223 \mu \mathrm{mol} / \mathrm{l}$ (reference: 60-100 $\mu \mathrm{mol} / \mathrm{l}$ ), urea was $17.7 \mathrm{mmol} / \mathrm{l}$ (reference: $2.5-6.5 \mathrm{mmol} / \mathrm{l}$ ), potassium was $4.1 \mathrm{mmol} / \mathrm{l}$ (reference: $3.5-5 \mathrm{mmol} / \mathrm{l}$ ), sodium was $138 \mathrm{mmol} / \mathrm{l}$ (reference: 135-145 mmol/l). Serum alanine aminotransferase was $10 \mathrm{IU} / \mathrm{l}$ (reference: 4-36 IU/l), serum aspartate aminotransferase was 14 IU/I (reference: 4-36 IU/I), serum alkaline phosphatase was 88 IU/I (reference: 45-146 IU/I), and serum bilirubin was $12 \mathrm{mmol} / \mathrm{I}$ (reference: 4-17 mmol/l). Anti-nuclear antibodies were positive at a titre of $1: 160$ (speckled pattern). Stool microscopy and culture yielded no growth of microorganisms. Screening of laboratory tests for viral diseases - hepatitis B and C viruses and human immunodeficiency virus - was negative. A serum ADAMTS13 test was planned but could not be done as the facility was not available in our setting. However, due to clinical symptoms and presence of schistocytes greater than $3 \%$ a presumptive diagnosis of TTP was made.

The patient was treated with a three-day course of intravenous pulse of methylprednisolone at $1 \mathrm{~g}$ daily and later maintained by oral prednisolone at $60 \mathrm{mg}$ daily (1 mg/kg). Knee joints were punctured with synovial fluid evacuation, and glucocorticosteroids were also administered to the joints. Two units of red blood cell concentrate were also transfused.

After the administration of the described treatment, a rapid improvement was observed. Urea and creatinine levels also normalised over a week after commencement of treatment. The patient was discharged after 10 days from admission, by which time the prednisolone had been stopped. PCV at the point of discharge was $32 \%$.
After the next two weeks the patient demonstrated tenderness in the wrists, metacarpophalangeal, and proximal interphalangeal joints of the hands as well as both knees. There was also effusion in both knees. In laboratory tests, electrolytes, urea and creatinine levels were normal. PCV was $24 \%$ with peripheral schistocytes but platelet and leucocyte counts remained within the normal range. There was no recurrence of the subcutaneous bleeding. The C3 and C4 complement components revealed a decrease of both components $-15 \mathrm{mg} / \mathrm{dl}$ (reference: $88-206 \mathrm{mg} / \mathrm{dl})$ and $2 \mathrm{mg} / \mathrm{dl}(12-75 \mathrm{mg} / \mathrm{dl})$, respectively. Due to the system of financing medical care in Nigeria, to a large extent the responsibility of the patient, in the described case it was not possible to perform more extensive diagnostic tests. However, based on the tests already carried out, it was possible to make a diagnosis. Taking into account the clinical symptoms of arthritis and laboratory results of ANA positivity and decrease of complement components, and previous haematological abnormalities such as thrombocytopenia, the diagnosis of systemic lupus erythematosus based on the Systemic Lupus International Collaborating Clinics (SLICC) criteria was established. It was decided to treat the patient with glucocorticosteroids (GCS) and hydroxychloroquine (HCQ) as well as introducing osteoporosis prophylaxis.

\section{Discussion}

Thrombotic thrombocytopenic purpura is an uncommon life-threatening disease which presents in approximately $0.5-2 \%$ of patients with SLE $[3,4]$. It was first described by Moschowitz in 1924, but the pentad of severe thrombocytopenia, microangiopathic haemolytic anaemia, neurologic abnormalities, renal insufficiency, and fever was established in 1966 by Amorosi and Ultmann [6, 7].

Our patient presented with the classical features of TTP stated above and exhibited a rapidly deteriorating disease course. The huge potential for misrecognizing TTP, a rare disorder, may be complicated by the complex picture of SLE starting out as TTP. While many patients with TTP in sub-Saharan Africa are at high risk of mortality due to unavailability of prompt diagnostic facilities and access to plasmapheresis, an African patient with TTP in SLE may be at an even greater risk. The presentation of a patient with TTP may not necessarily compel a clinician to work up for a primary connective tissue disease, including SLE, as both diseases can have overlapping features. In our patient, TTP was the first presentation of SLE, a finding which is contrary to previous reports that TTP usually presents in patients previously diagnosed and treated for SLE for several years, with a high SLE disease activity index score and coexisting nephritis [5, 8]. 
Polyarthralgia, arthritis, fever, hair loss, osteonecrosis and myopathy are common presentations reported in previous studies of patients with SLE and TTP [2, 9, 10]. However, TTP was the heralding manifestation in our patient, and it took a high index of suspicion spurred by the emergence of polyarthritis to pursue a complement assay, an immunologic finding that reinforced the diagnosis of SLE. The absence of the more common manifestations of SLE may delay the diagnosis and treatment of SLE, and the predominance of a rare manifestation such as TTP may lead to a diagnostic dilemma. The clinical overlap between these two syndromes has been described more commonly in young black women [11], and since certain severe manifestations have been found more commonly in SLE patients of African descent, TTP may constitute another such manifestation with notable links with genetic attributes of the patient.

Thrombotic thrombocytopenic purpura has been linked to diarrheal disease, which occurs in up to $15 \%$ of the patients after infection with toxin-producing Escherichia coli or Shigella dysenteriae [12]. Even though our patient had several episodes of diarrhoea prior to presentation, the stool culture yielded no microorganisms.

Although plasma exchange continues to be the mainstay of treatment in patients with TTP, even with concomitant SLE, this option is often lacking in hospitals in third world countries. Whereas plasmapheresis is not an important treatment option for lupus, the similar mechanistic function of intravenous immunoglobulin can probably offer useful intervention when severe TTP complicates SLE. Importantly, both TTP and SLE are often treated with high doses of intravenous methylprednisolone. In unclear situations, and obviously in resource-poor settings, this treatment may be a reliable option. Our patient was treated with intravenous methylprednisolone with a good result, and plasmapheresis in the course of TTP was not necessary. Glucocorticosteroids are a common treatment component in many severe manifestations of SLE.

Furthermore, the high frequency of occurrence of neurologic complications in SLE patients of African descent may cause difficulty in diagnosis and proper treatment. Pathophysiologically, the deficiency of the enzyme ADAMTS13 metalloproteinase giving rise to TTP is usually due to autoantibody production and activity. As a result, low levels of ADAMST13 are common to all forms of TTP but the presence of lupus-specific immunological markers such as anti-Sm and anti-dsDNA antibodies is important to guide the identification of SLE. Logically, therefore, the lupus patient with TTP will not only be almost always positive for anti-nuclear antibody, there is a good chance of finding low components of complement or other lupus-associated antibodies. This pattern is less likely in isolated TTP cases.

\section{Conclusions}

The association of SLE and TTP is uncommon but potentially lethal even with current treatment strategies. Early diagnosis and aggressive management with glucocorticosteroids and other immunosuppressive medications, and in certain cases plasma exchange therapy, is the key. It is not always possible to perform all immunological tests, but it is possible to diagnose both TTP and SLE based on available knowledge and essential immunological markers. Some limitations in immunological tests should not delay diagnosis.

Critical care specialists, rheumatologists, and haematologists need to evolve an urgent multidisciplinary approach which emphasizes the early recognition of this phenomenon and the initiation of early treatment which can secure a better outcome.

The authors declare no conflict of interest.

\section{References}

1. Jessica JM, Anisur R. Systemic lupus erythematosus. Orphanet J Rare Dis 2006; 1: 6.

2. Adelowo OO, Oguntona SA. Pattern of systemic lupus erythematosus among Nigerians. Clin Rheumatol 2009; 28: 699-703.

3. Tsai HM. Pathophysiology of thrombotic thrombocytopenic purpura. Int J Hematol 2010; 91: 1-19.

4. Musio F, Bohen EM, Yuan CM, et al. Review of thrombotic thrombocytopenic purpura in the setting of systemic lupus erythematosus. Semin Arthritis Rheum 1998; 28: 1-19.

5. Shah AA, Higgins JP, Chakravarty EF. Thrombotic microangiopathic hemolytic anemia in a patient with SLE: diagnostic difficulties. Nat Clin Pract Rheumatol 2007; 3: 357-362.

6. Amorosi EL, Ultman JE. Thrombotic thrombocytopenic purpura: report of 16 cases and review of the literature. Medicine 1966; 45: 139-159.

7. Lämmle B, Kremer Hovinga JA, Alberio L. Thrombotic thrombocytopenic purpura. J Thromb Haemost 2005; 3: 1663-1675.

8. Kwok SK, Ju JH, Cho CS, et al. Thrombotic Thrombocytopenic purpura in systemic lupus erythematosus: risk factors and clinical Outcome: a single centre study. Lupus 2009; 18: 16-21.

9. Maddison PJ. Is it SLE? Best Pract Res Clin Rheumatol 2002; 16: 167-180.

10. Zoma A. Musculoskeletal involvement in systemic lupus erythematosus. Lupus 2004; 13: 851-853.

11. Terrell DR, Vesely SK, Hovinga JAK, et al. Different disparities of Gender and race among the thrombotic thrombocytopenic purpura and hemolytic-uremic Syndromes. Am J Hematol 2010; 85: 844-847.

12. Tsai H-M. Thrombotic Thrombocytopenic Purpura: A Thrombotic Disorder Caused by ADAMTS13 Deficiency. Hematol Oncol Clin North Am 2007; 21: 609. 